\title{
QUELQUES DONNÉES SUR LA PÊCHE AMATEUR EN POLOGNE
}

\author{
B. BRETON*, R. BILLARD ${ }^{* *}$
}

La Pologne compte en 1978 quelques 750000 à 800000 pêcheurs amateurs sur une population de 35000000 d'habitants, soit $2.3 \%$ de la population, alors que le chiffre atteint $9 \%$ environ en France. Leur nombre croit rapidement puisque l'on a enregistre 150000 adhérents de plus, entre 1976 et 1978, aux associations de pêcheurs sportifs $(P Z W)$. Cependant ce chiffre officiel concerne les pêcheurs dits légaux, c'est-à-dire titulaires d'une licence annuelle, mais si l'on inclut des pêcheurs semi-légaux (permis temporaires et illégaux, braconniers), le nombre total de pêcheurs serait de l'ordre de 2 millions

Les raisons de l'augmentation rapide du nombre de pêcheurs sont nombreuses et sont essentiellement lièes à l'urbanisation rapide, à l'augmentation du parc automobile, à l'apparition sur le marché polonais d'un matériel de qualité. II faut noter à ce sujet que le matériel français est le plus apprécié des pêcheurs polonais. Enfin, outre le divertissement qu'offre la pratique de ce sport, les dirigeants du PZW reconnaissent qu'une partie de leurs adhérents recherche une source de protéines animales pour suppléer à la crise très grave que rencontrent actuellement la production et la distribution du secteur agro-alimentaire. Cependant, de nombreux pêcheurs recherchent avant tout le loisir, et c'est essentiellement l'aspect sportif qui attire le pêcheur de salmonidés

Les pêcheurs sont groupes localement en associations (42) qui couvrent des zones administratives, lesquelles sont au nombre de 49. C'est-à-dire que tous les "départements" ne sont pas encore tous pourvus d'association de pêcheurs. Cela est dû au faible nombre de pêcheurs dans certaines régions et au fait qu'il faut au moins 3000 membres pour creer une association. Les associations des pêcheurs sont groupées dans une structure nationale $(P Z W)$ qui a son siège à Varsovie. II y a en outre 12 institutions techniques régionales. Elles sont différentes des anciennes subdivisions administratives et se sont vu confier la responsabilité des plans d'eau dans leur intégralité. Les attributions de ces

- Laboratoire de Physiologie des Poissons. INRA. Campus universitaire de Rennes-Beaulieu. BP 25 A 35031 RENNES CEDEX

** Laboratoire de Physiologie des Poissons. INR.A. 78350 JOUY.EN-JOSAS.

Article available at http://www.kmae-journal.org or http://dx.doi.org/10.1051/kmae:1980004 
institutions régionales sont essentiellement la gestion des stocks piscicoles et des piscicultures de repeuplement. Elles disposent de moyens materiels (materiel de pêche, de transport) mis à la disposition des associations locales. Par contre, elles n'ont pas autorité de décision en ce qui concerne la politique de repeuplement; dans ce domaine elles ne font quexecuter les décisions prises au niveau national. Ceci aboutit à une politique de repeuplement globale, qui, daprès nos interlocuteurs, donne de bons résultats et élimine les abus dûs aux déversements ponctuels réalıses la plupart du temps avec finalité politique. Ces associations régionales ont, cependant, la responsabilité de surveillance et entretien des plans d'eau. Au sujet de la surveillance des plans d'eau, il faut noter qu'outre les gardes assermentes au nombre de 600. il existe un corps de volontaires (environ 1000 ) habilitès à verbaliser. L'association nationale des pêcheurs organise des reunions avec des chercheurs de diverses origines afin de faire le point des connaissances dans les différents domaines, et de définir ainsi sa politique d'ensemble et se forger une doctrine pour ses actions techniques C'est ainsi quiun symposium en 1973 a réuni 100 participants avec présentation de 5 communications de synthèse et 15 exposés scientifiques portant sur l'aménagement, l'exploitation et la protecton des eaux et de ieurs ressources. la prévision dans lexploitation. ainsi que les aspects légaux et socio. économiques de la pêche sportive.

Les besoins de la pêche amateur sont souvent defınis d'après le type d'activité de pêche et le poisson correspondant, mais aussi d'après les types d'aménagement de récréation à mettre en place (considerés du point de vue quantitatif et qualitatif, cf. tabl. 1)

Tableau 1 : Les besoins en aménagements loisirs selon les types de pèche pratiqués et les espèces favorites des pêcheurs amateurs (d'après LEOPOLD, 1977, EIFAC/T 26, 46-62).

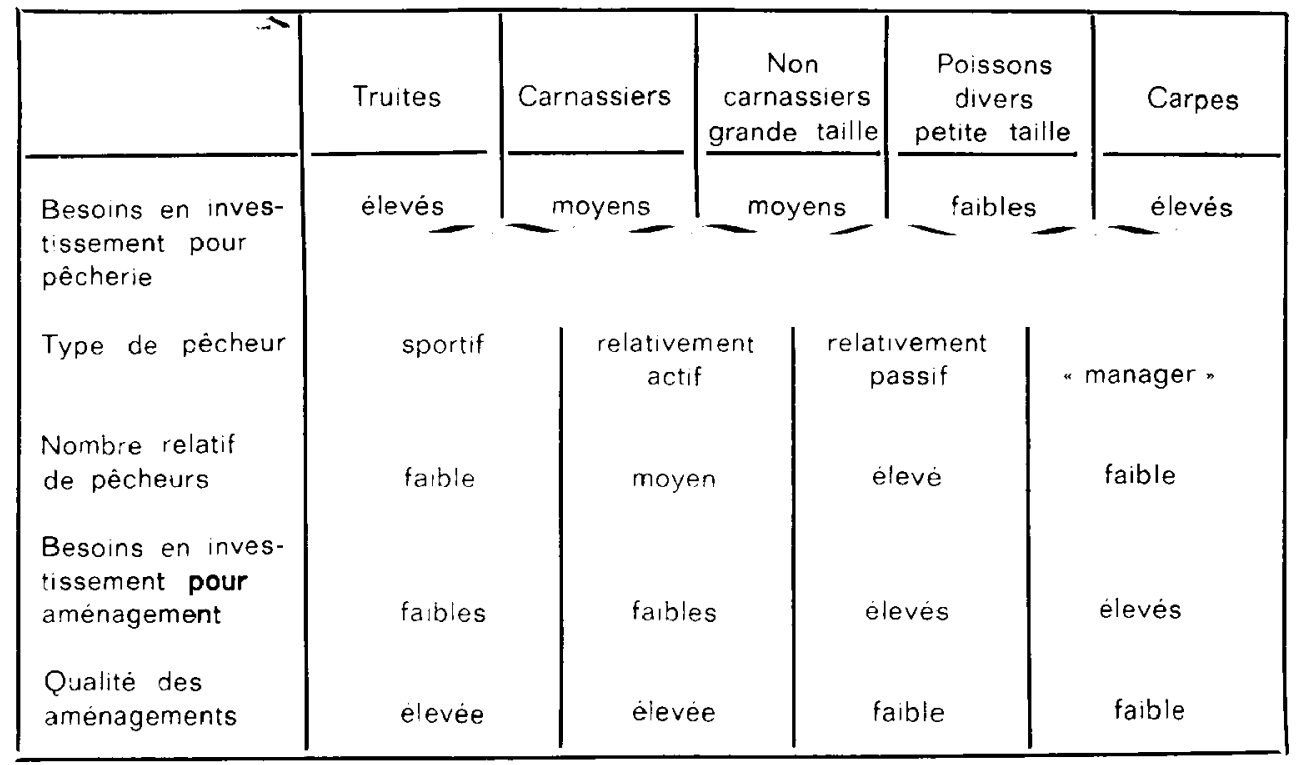

Sur les 400000 ha d'eau douce susceptibles de faire l'objet de pêche amateur. lassociation des pêcheurs sportifs nien contrôle que 240000 (40\% des eaux du territoire polonais). En outre, $\mathrm{PZW}$ assure l'amenagement et la gestion directe avec exploitation commerciale de 78000 ha deau et possède 43 centres touristiques comportant des logements pour 1000 personnes Lassociation organise en nutre des competitions ayant Suni 185000 personnes en 1974 et des excursions de pêche (10000 concernant 240000 personnes en 1974). En plus lactivité de production de poisson pour le repeuplement (5 kg de poisson produits/ha d'eau contrôlee) est importante (cf. ci-après) La majeure 
partie du domaine pêchable est constituée par lensemble des rivières, petits iacs, etangs, et les lacs de Mazurie, les rivières du sud et de Poméranie fournissent les aires de pêche des saimonidés. Dans les rivières if n'y a pas compétition entre pêcheurs professionnels et sportifs, car il n'existe pratiquement plus de pêche commerciale sur les

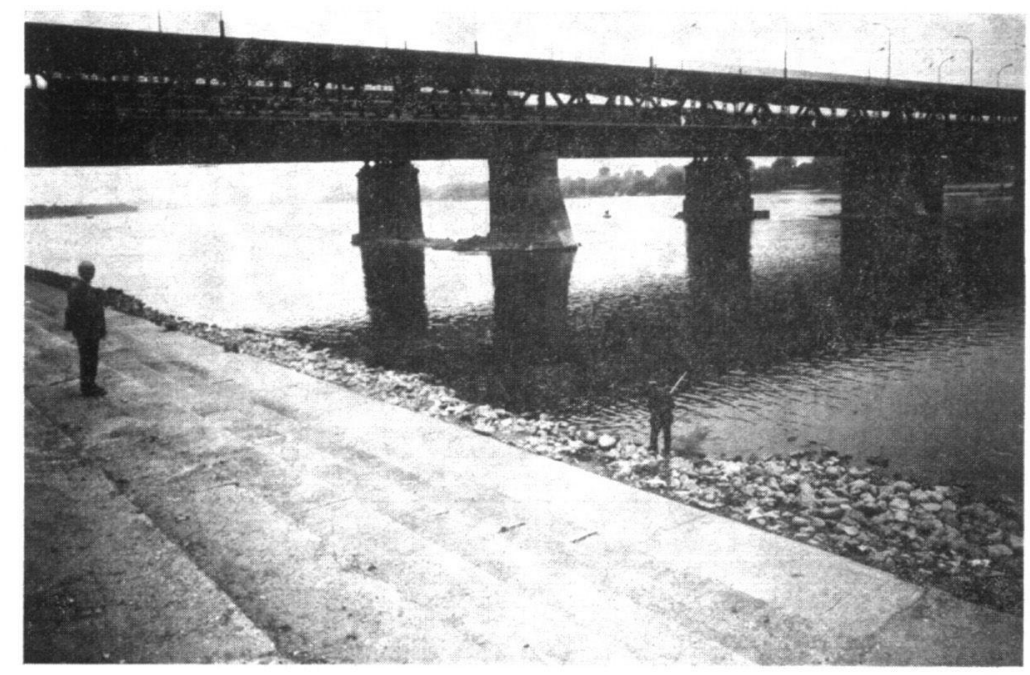

Photo 1 : Pêcheurs sur les bords de la Vistule à Varsovie.

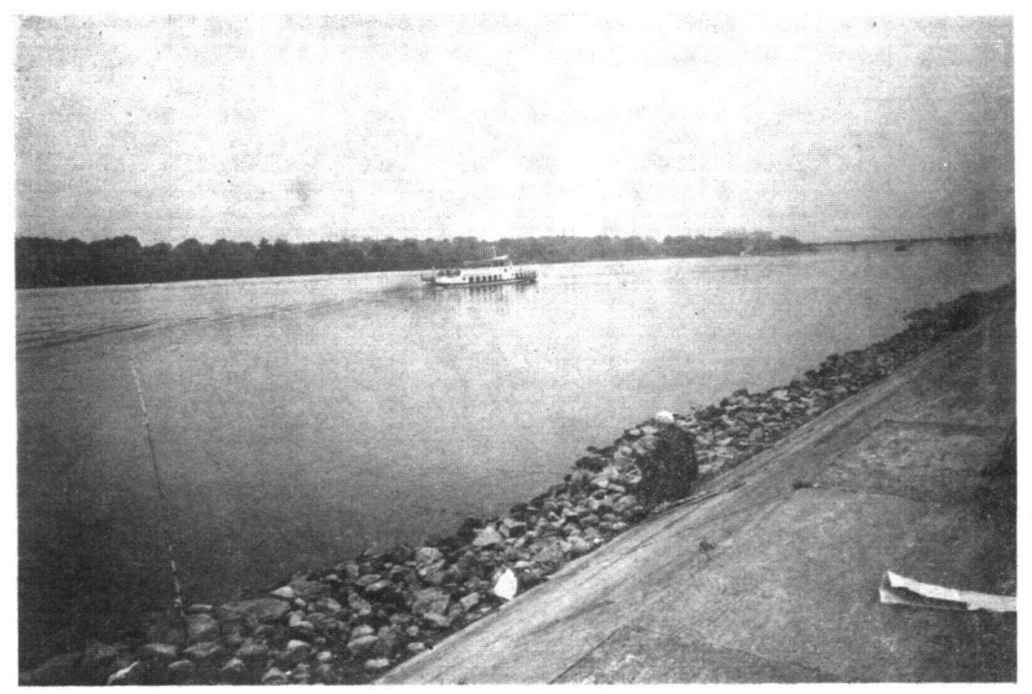

Photo 2 : Pêche à l'aide d'un petit carrelet sur les bords de la Vistule à Varsovie. 
rivières polonaises (photos 1-2) Seules quelques cooperatives poursuivent une activite de cueillette sur la partie ava! do la Vistule (barrage de la Vistule bouche de la Vistu' et golf cu Gdanz). Il y a par contre competition dans les lacs entre pêch a professionnelí et péche amateur. Environ $60 \%$ des eaux gérees pa: PZW iont egalement l'objet de pêche par des pécheries d'Etat. II y a bien sûr conflit dinterêt. mass h' a etè demontre que ce sont surtout les pêcheurs semi-legaux ou illegaux qui exercent un impact négatif de sorte que les pécheurs légaux et leur association, qui du fait du gardiennage limiten: le braconnage, cohabitent aisement avec les pêcheurs professionneis. Des convergences d'intérêt se trouvent aussi dans la lutte contre la pollution Les captures par les pêcheurs toutes catégories ne sont cependant pas néglıgeables et atteignent $14 \mathrm{~kg} / \mathrm{ha}$ an pêcheur. soit $40 \%$ de celies des pêcheurs professionnels (selon les regions ce pourcentage varie entre 30 et $80 \%$ ). LEOPOLD (1977. EIFACT26,p. 46-62) a calcule que $80 \%$ de ces prises sont le fait de pêcheurs locaux. II faut soulignei cette cohabitation pacifique entre ces deux activités qui entrent en competition. les attitudes extrémes consistant à exclure le pêcheur amateur ou à considerer que la pêche professionnelle limite les captures des amateurs. La coexistence de ces deux types dactivite revient probablement à augmenter la productivité de ces plans deau non vidangeables

Les moyens financiers de lassociation sont exclusivement constitués par les cotisations des membres. Ces dernières sont de 250 Zlotys pour la licence de base autorisant la pêche des cyprinides, 400 Zlotys si les salmonidés y sont inclus. II existe des taxes spéciales pour certaines espèces (brochet), certains sites (lacs) ou pour certains moyens de pêche (par exemple le droit de péche en las. en bateau, coûte 10 fois pius cher que celui de la pêche en bordure.

\section{LES MOYENS DE REPEUPLEMENT (statistiques arrêtées fin 1976)}

Une des caractéristiques générales de la politique de repeuplement porte surtout sur le lâcher d'alevins. En 1976, 550 tonnes de poissons (toutes espèces confondues) ont été déversées, dont 329 produites par les piscicultures de l'association des pêcheurs, le reste est acheté à des fermes piscicoles de l'Etat

Les structures de production représentaient, en 1976, 655 ha. Dans toutes les piscicultures, le directeur de l'établissement a suivi un enseignement ichtyologique, et les plus grosses unites sont dirigees par un ingénieur ichtyologue. L'équipement des unités de production est très variable mais, la plupart du temps, il est de bonne qualité et complet (ains! la plupart possèdent une chambre froide de conservation des aliments). Un souci particulier est porté au contrôle sanitaire des élevages

De nombreux plans deau sont actuellement surexploites, principalement en régions touristiques et en zones urbanisees. Les captures sont elevees : $50 \mathrm{~kg}$ pècheur an alors que la production naturelle aménagée n'est que de quelques dizaines de $\mathrm{kgha}$ h. Les responsables ont réussi à conduire un aménagement rationnel des divers plans d'eau, basé sur un repeuplement par des juvéniles qui présentent un taux de survie acceptable Laugmentation de la pression de pêche pose un probleme difficile aux gestionnaires de la pêche. Il serait logique d'encourager le pêcheur à se déplacer en dehors des périmètres urbanisés où la pression de pêche est moins forte, mais les difficuitès de communications font que les personnes âgées se verront privées de cette activité

La pêche amateur porte surtout sur les cyprinidés, salmonides et diverses espèces comme le brochet, le sandre et languille.

\section{LES CYPRINIDES}

Deux types de produrtion sont assurés :

1) Stocks naturels constitués des étangs, lacs naturels et de barrage Dans cette catégorie, lacs et étangs entrent pour 17,4 ha. les lacs de barrage étant exploités sur 78 ha 
2) Piscicultures classiques : tassociation posseje actuellement 29 établissements classes selon leur surface d'exploitation

$\begin{array}{llrl}\text { Superficie infèrieure à } 20 \text { ha } & 18 \text { etablissements } & 193 \text { ha } \\ \text { Superficie comprise entre } 20 \text { et } 40 \text { ha } 7 \text { établissements } & 202 \text { ha } \\ \text { Superficie supérieure à } 40 \text { ha } & 4 \text { établissements } & 235 \text { ha }\end{array}$

A titre d'exemple, ces piscicultures ont produit en 1976

225 tonnes de carpe commune et sauvage :

2.3 tonnes de barbeau, Ide et Vimba vimba;

20,2 tonnes de brochet et sandre;

188 tonnes de brème, gardon, rotengle et tanche

Ces structures paraissent d'ores et déjà insuffisantes, une augmentation de leu capacité et de leur rendement est recherchee par utilisation de système en eau rechauffée

\section{LES SALMONIDES}

La capture des salmonidés nécessite l'achat d'un timbre spécial qui n'est acquis que par $6 \%$ de l'ensemble des pêcheurs. Par contre. l'effort de repeuplement realisé en faveur de ces quelques 4500 personnes est considérable, puisque 23 piscicultures leur sont consacrées, qui ont produit 35,8 tonnes de poissons, soit 4079000 alevins et 200800 smolts (truite de mer seulement), soit $60 \%$ des smolts de truite de mer lâchés en Pologne

Les espèces produites sont les suivantes:

Truite arc-en-ciel

Truite fario

Omble de fontaine

Ombre

Truite de lac

Saumon du Danube (Hucho hucho)

Truite de mer

Saumon atlantique

La production varie fortement entre ces différentes espèces. La truite de mer est l'espèce la plus importante et plus de $90 \%$ des captures de salmonidés sont représentés par cette espèce. Le saumon atlantique n'est produit que dans une seule rivière (Drava); le taux de capture pour cette espèce est estimè à $2,5 \%$, mais est surtout le fait de pêche commerciale en mer, les captures par pêcheur sportif ne présentant que $0,8 \%$ du total. Les difficultés techniques de production varient aussi avec les espéces. Le saumon atlantique est l'espèce dont l'élevage est le plus difficile, suivi par la truite de mer, mais les techniques commencent à s'affiner; au moins pour cette dernière et dans les meilleurs des cas, on atteint des survies œuf-smolt à 2 ans de $50-60 \%$. L'omble est aussi une espèce d'élevage difficile; il n'existe que 2 à 3 écloseries. Les géniteurs sont capturés sur les zones de fraie, transportés dans les écloseries. Les œufs après insémination sont mis en incubateurs californiens. La première prise alimentaire est délicate Les aliments de démarrage, de type Ewos ou autres, doivent être complementés par du foie ou de la rate additionné de vitamines. La première alimentation est aussi le point critique pour Hucho hucho. En effet, cette espèce n'accepte que les proies vivantes, et si les chercheurs polonais ont réussi en 1977 à boucler un cycle en captivité avec de la nourriture artificielle, les produits sexuels obtenus se sont averés non fécondables. Un parc de géniteurs de plusieurs tonnes est conservé pour la plupart de ces espèces dans les écloseries. 
Depuis 4 ans, il est fait obligation de marquer au moins $1 \%$ des animaux lors de tout lâcher. De ce fait. les taux de recapture sont suivis en permanence pour toutes les espèces. Ils s'èlèvent globalement de 15 à $16 \%$ dont :

$64,87 \%$ pour la truite fario

$10.74 \%$ pour la truite arc-en-ciel

Taille limite

$25 \mathrm{~cm}$

$3.69 \%$ pour lomble de fontaine

$30 \mathrm{~cm}$

$1.04 \%$ pour la truite de mer

$0.01 \%$ pour le saumon du Danube

$50 \mathrm{~cm}$

$60 \mathrm{~cm}$

Les pourcentages obtenus sur la truite de mer sont certainement sous-estimes. En effet, parallèlement aux déversements réalisés par l'association des pêcheurs, d'autres alevinages sont faits par des fermes d'Etat à fin de * sea-ranching . Cette activite s'exerce principalement par pêche au filet directement dans la Baltique. De ce fait, nombre de smolts relâchés par l'association des pêcheurs sportifs doivent être repris par la péche professionnelle. En 1975-1976, on considère que les pêcheurs de salmonidés ont pêché en moyenne 16,5 jours:an, pour des prises de 15,7 poissons, soit 1 poisson jour personne. Outre les tailles légales à respecter et les perriodes d'ouverture. il existe des limitations sur le nombre de prises autorisees selon les espèces : 5 jour pour truites et ombres, 2 jour pour la truite de mer, 1 semaine pour le saumon du Danube. Outre ces deux principales espèces, il existe également une pression de pêche importante sur d'autres espèces, tels les corrégones (Albula et lavaretus) specialement dans les lacs de Mazurie.

\section{LES AUTRES ESPECES}

Le brochet est une espèce très prisée par les pêcheurs, mais dont l'importance diminue dans les rivières du fait de la pression de pêche, de la canalisation de ces rivières qui entraine la suppression des zones inondables où le brochet vient se reproduire. Les problemes de pollution peuvent aussi contribuer à réduire les populations de brochet. Quelques écloseries-nourriceries pratiquent des productions d'œufs embryonnés ou alevins
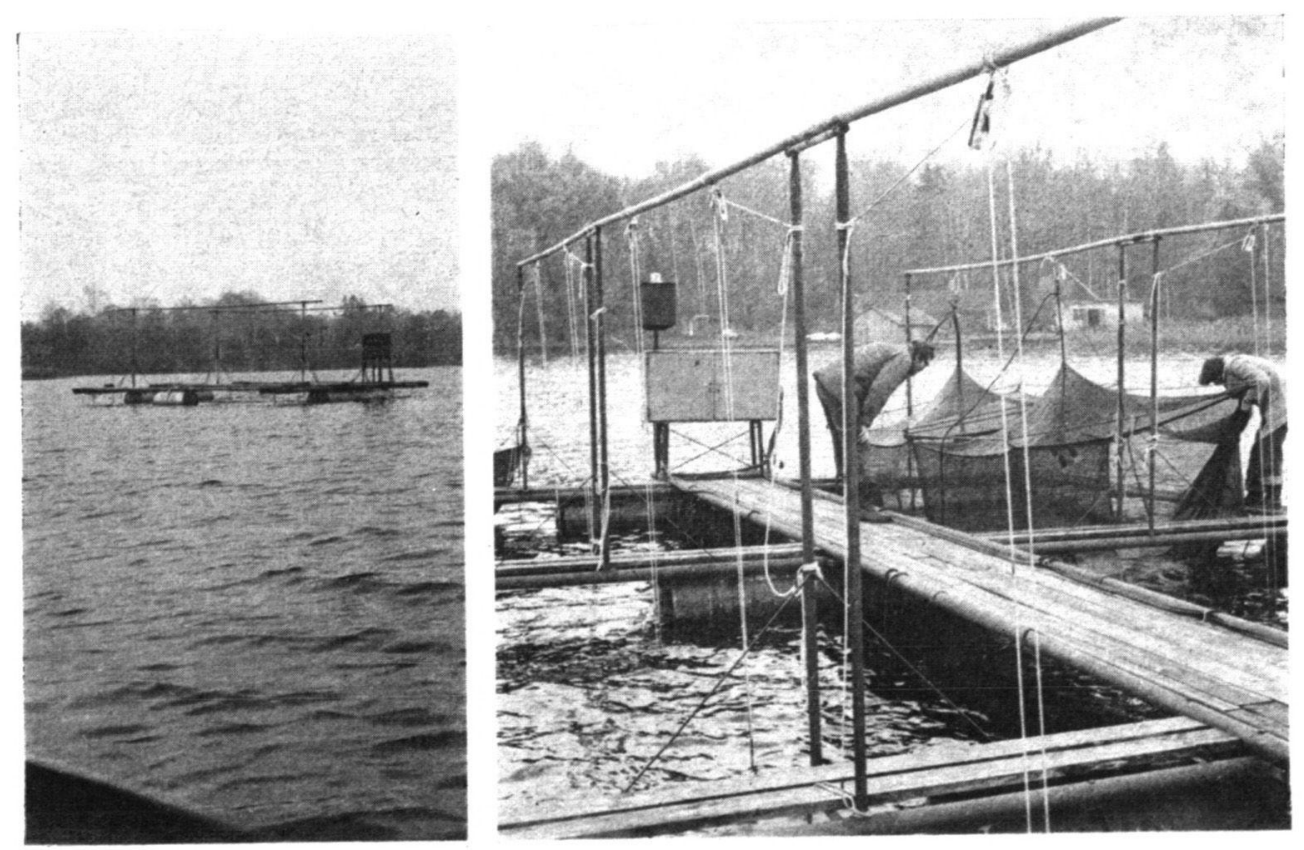

Photo 3 : Elevage de jeunes coregones en cages sur un lac du nord de la Pologne; à gauche batterie de cages, vue gènérale; à droite détail du dispositif permettant de soulever les cages. 
a resorption pour le repeuplement. Generalement, on pratique fincubation dans les bouteilles de Zug des écloseries à coregones. Les femelles ovulees sont capturées dans des nasses lors de leur remontée sur les frayères ou bordures de lacs. Une partie des œufs embryonnés est exportée. Il existe quelques tentatives d'élevage des juveniles en cage immergeee, en lac, basées sur l'attraction du plancton par lampe électrique, comme cela se pratique pour les corégones (photo 3 ).

La production danguilles est tres particuliere en Pologne. II n'y a pas de recrutement naturel et un repeuplement est pratique par les services gouvernementaux a partir d'elvers importees de France ou d'Allemagne. Les captures des pêcheurs amateurs ne sont pas connues. mais cel!es des pêcheurs professionnels sont de l'ordre de $800 \mathrm{~T}$ an

\section{CONCLUSION}

La pêche amateur est un divertıssement de plus en plus popularre en Pologne. "I est encore trop tôt pour se prononcer sur les effets de la reorganisation de l'association des pêcheurs qui a eu lieu il y a douze ans, mais il est à noter que comme dans le cas de la chasse, la pêche dispose dune incontestable autonomie financière. ce qui luı confere une certaine liberté d'action Cela resulte très probablement du falt que la chasse et la pêche (photo 4) sont libres depuis très longtemps dans ce pays.

\section{REMERCIEMENTS}

Cette note a ete rédigee dans ie cadre des collaborations CSPINRA Nous remercions nos collègues polonais qui ont organise nos déplacements, et avec lesquelo nous avons eu de fructueuses discussions (Drs. WIENIAWSKI, GORICZKO et LEOPOLD) 


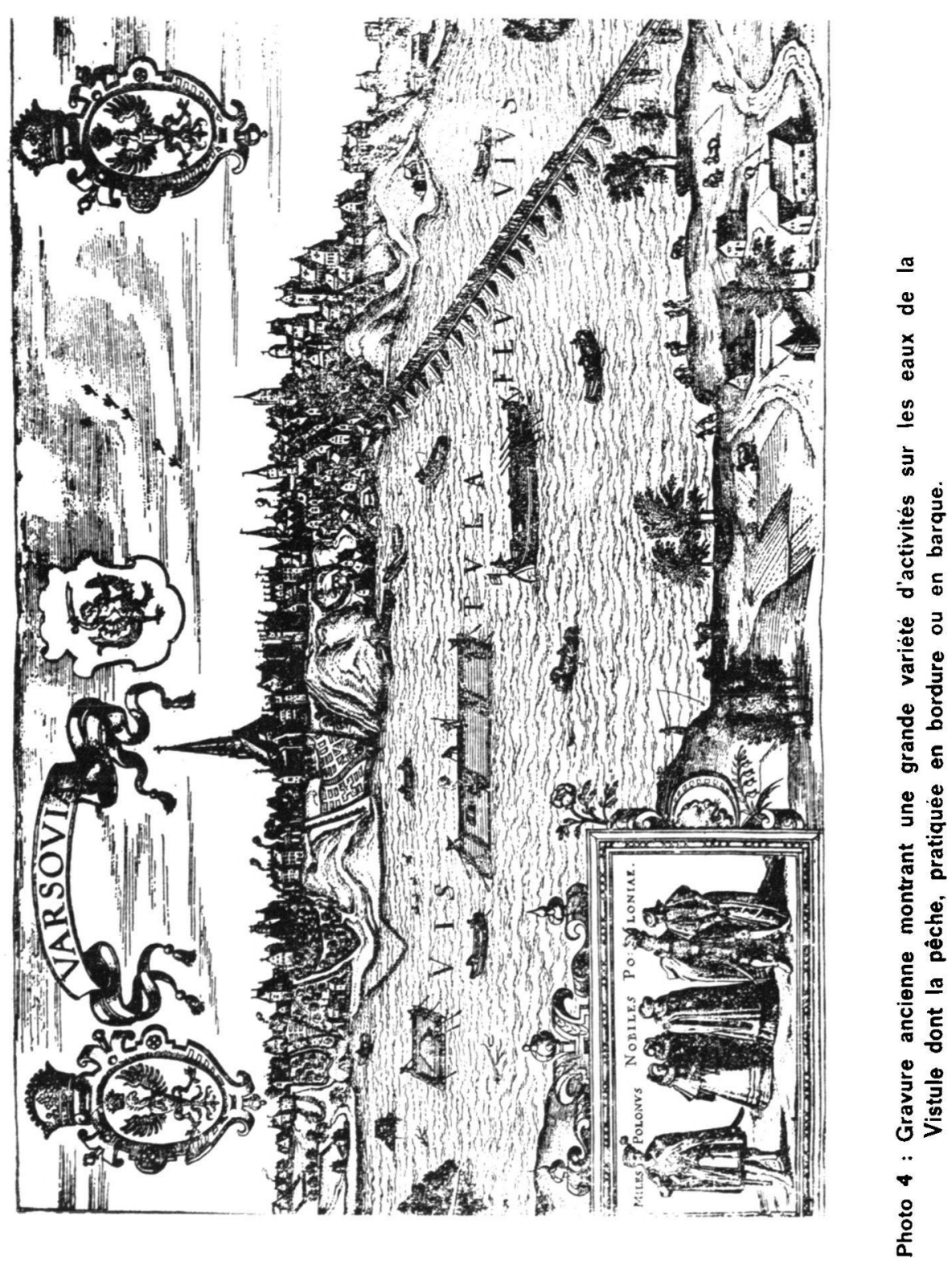

